\title{
Multi Objective Evolutionary Algorithm Applied to the Optimal Power Flow Problem
}

\author{
E. A. Amorim, S. H. M. Hashimoto, F. G. M. Lima, Member IEEE and J. R. S. Mantovani, Member IEEE
}

\begin{abstract}
This work presents the application of a multiobjective evolutionary algorithm (MOEA) for optimal power flow (OPF) solution. The OPF is modeled as a constrained nonlinear optimization problem, non-convex of large-scale, with continuous and discrete variables. The violated inequality constraints are treated as objective function of the problem. This strategy allows attending the physical and operational restrictions without compromise the quality of the found solutions. The developed MOEA is based on the theory of Pareto and employs a diversity-preserving mechanism to overcome the premature convergence of algorithm and local optimal solutions. Fuzzy set theory is employed to extract the best compromises of the Pareto set. Results for the IEEE-30, RTS-96 and IEEE-354 test systems are presents to validate the efficiency of proposed model and solution technique.
\end{abstract}

Keywords - Multiobjective Evolutionary Algorithm, Optimal Power Flow, Multiobjective Optimization.

\section{NOMENCLATURA}

$i$ e $j$

: Índices das barras e geradores.

$k$ e $l$

$N$ e $G$

: Índices das funções objetivo.

: Conjuntos de barras e de geradores do sistema, respectivamente.

$C_{2 i}, C_{1 i}$ e $C_{0 i} \quad$ : Coeficientes de custo do $i$-ésimo gerador.

$P_{g i}$ e $Q_{g i}$

: Potências ativa e reativa gerada pelo $i$-ésimo gerador.

$P_{L i}$ e $Q_{L i}$

: Potências ativa e reativa demandada pela $i$ ésima barra.

$P(V, \theta, t)_{i} \quad$ : Injeção líquida de potência ativa na barra $i$.

$Q(V, \theta, t)_{i} \quad:$ Injeção líquida de potência reativa na barra $i$.

$P_{g i}^{\min }$ e $P_{g i}^{\max } \quad$ : Capacidades mínima e máxima da geração de potência ativa do i-ésimo gerador.

$Q_{g i}^{\min }$ e $Q_{g i}^{\max } \quad$ : Capacidades mínima e máxima da geração de potência reativa do i-ésimo gerador.

$V_{i}$ e $\theta_{i} \quad:$ Magnitude de tensão e ângulo de fase na barra $i$

$t_{i} \quad: i$-ésimo transformador com controle automático de taps.

$S_{H_{i}} \quad:$ Banco de capacitor/reator shunts na $i$-ésima

Os autores agradecem a FUNDECT e CNPq (Processo 350226/2007-5) pelo financiamento deste projeto de pesquisa.

E. A. Amorim, S. H. M. Hashimoto e F. G. M. Lima pertencem ao Depto. de Engenharia Elétrica, Universidade Federal de Mato Grosso do Sul - UFMS, Campo Grande - MS - Brasil, elizete.amorim@gmail.com; selma_hashimoto@yahoo.com.br; flavio@del.ufms.br.

J. R. S. Mantovani pertence ao Depto. de Engenharia Elétrica, UNESP, Ilha Solteira - SP, Brasil, mant@dee.feis.unesp.br.

\author{
barra. \\ $V_{i}^{\min }$ e $V_{i}^{\max } \quad$ : Limites inferior e superior da magnitude de \\ tensão na i-ésima barra. \\ $S_{i j} \quad:$ Fluxo de potência aparente sobre a linha $i j$. \\ $S_{i j}^{\max } \quad:$ Capacidade máxima de fluxo potência \\ aparente sobre a linha $i j$. \\ $t_{i}^{\min }$ e $t_{i}^{\max } \quad:$ Limites inferior $\mathrm{e}$ superior do $i$-ésimo \\ transformador com controle automático de \\ taps. \\ $S_{H_{i}}^{\min }$ e $S_{H_{i}}^{\max }$ : Limites inferior e superior do \\ capacitor/reator shunts na $i$-ésima barra. \\ $P_{g_{\text {ref }}}$ e $P_{g_{\text {ref }}}^{\max }$ \\ Potência ativa gerada e capacidade máxima \\ $\Delta \mathbf{u}_{D}$ \\ $u_{D_{i}}^{\min }$ e $u_{D_{i}}^{\max }$ \\ de geração de potência ativa na barra slack. \\ : Tamanho do passo de discretização. \\ $\operatorname{Pr}^{\min }$ e $\operatorname{Pr}^{\max }$ \\ Limites \\ de recombinação. \\ $\mathrm{Pm}^{\min }$ e $\mathrm{Pm}^{\max } \quad$ : Limites inferior e superior referentes à taxa \\ de mutação. \\ ig e nmax : Índices e número máximo de gerações. \\ $f_{k}^{\min }(x) \quad$ : Valor mínimo da $k$-ésima função objetivo. \\ $f_{k}^{\max }(x) \quad$ : Valor máximo da $k$-ésima função objetivo.
}

\section{INTRODUÇÃO}

TESTE trabalho destaca-se a aplicação dos algoritmos evolutivos multiobjetivo (AEMO) para a solução do problema de fluxo de potência ótimo (FPO). A aplicação de técnicas de otimização no planejamento e operação dos sistemas elétricos de potência se justifica devido ao fato de que é altamente vantajoso para as concessionárias de energia elétrica operarem seus sistemas de uma maneira ótima, ou pelo menos otimizada o quanto seja possível, para atender os critérios técnicos de segurança e redução de custos dos investimentos e operação.

O FPO [1] representa uma vasta classe de problemas que na área de planejamento de sistemas elétricos de potência buscam otimizar uma ou mais função objetivo enquanto satisfaz um conjunto de restrições físicas e operacionais. Sob o aspecto da pesquisa operacional, trata-se de um problema de otimização combinatorial complexo de grande porte que possui características como não-linearidade, não convexidade, várias restrições e variáveis discretas. Além destas características, o FPO apresenta múltiplas soluções locais e restrições nãolineares e descontínuas o que torna o problema um desafio para os pesquisadores da área de planejamento e operação de 
sistemas elétricos.

$\mathrm{Na}$ literatura especializada encontram-se diferentes técnicas para a solução do problema de FPO. Dentre estas técnicas destacam-se as metodologias convencionais tais como programação linear, programação não-linear, relaxação lagrangeana, métodos de pontos interiores, programação quadrática, entre outras [2-4]. O sucesso destas técnicas depende da convexidade do problema, mas devido à natureza do problema de FPO (não-convexo e não-diferenciável) os algoritmos baseados nessas hipóteses não guarantem encontrar o ótimo global. Portanto, os métodos convencionais que fazem uso de derivadas não são, em geral, capazes de localizar ou identificar o ótimo global. Adicionalmente, para a aplicação desses métodos são necessárias algumas hipóteses como funções objetivo diferenciável e convexa. Salienta-se, ainda, que nestes métodos os resultados obtidos dependem das aproximações ao ótimo considerado no início do processo iterativo e, que eles não são eficientes na resolução de problemas com espaços de busca de natureza discreta. Desta forma, torna-se indispensável o desenvolvimento de técnicas de otimização capazes de superar estes inconvenientes e tratar com eficiência tais dificuldades.

Em contraste com os métodos convencionais, os Algoritmos Evolutivos (AEs) [5] iniciam a busca de ótimos locais ou global a partir de uma população de soluções potenciais gerada de forma aleatória e guiam a procura com base em mecanismos e/ou regras probabilísticas. Estes algoritmos não utilizam nenhuma informação referentes às primeiras e/ou segundas derivadas da função objetivo e/ou restrições e podem utilizar mecanismos que permitem encontrar múltiplos ótimos locais (se existirem) numa única execução. Estas características fazem com que estes algoritmos sejam particularmente eficientes na resolução de muitos problemas reais, em que o espaço de busca muitas vezes contém um elevado número de ótimos locais e/ou mais de uma solução ótima. Por outro lado, são capazes de resolver problemas que apresentam espaço de busca discreta onde não é possível garantir as condições de diferenciabilidade e continuidade.

No entanto, quando o problema de otimização é restrito, caso do problema de FPO, os operadores originais de busca dos AEs não garantem a obtenção de soluções viáveis. Estudos sobre o desenvolvimento e uso de AEs para a solução de problemas restritos são recentes e de acordo com [6], a solução de problemas desta classe limita o uso dos AEs em sua forma original, principalmente pelo fato de não existir a garantia de que a factibilidade será mantida após a recombinação ou mutação.

$\mathrm{Na}$ literatura existem alguns procedimentos que podem ser adotados para tratar os problemas restritos, tais como: técnicas de penalização, reparação de soluções inviáveis, tratamento por múltiplos objetivos, operadores genéticos modificados e alterações na formulação do problema [7], [24].

Neste trabalho, as restrições de desigualdade violadas são tratadas como funções objetivo do problema. Desta forma, o problema original é transformado em um problema de otimização multiobjetivo que atende as restrições físicas e operacionais sem comprometer a qualidade das soluções encontradas. A solução do problema é determinada através de um algoritmo evolutivo multiobjetivo (AEMO) baseado na teoria de Pareto [8-10]. O AEMO explora uma estratégia elistista para preservar as soluções não-dominadas encontradas e um mecanismo de preservação da diversidade para evitar a convergência prematura do algoritmo e soluções ótimas locais. Além disso, um procedimento de desacoplamento implícito das variáveis de controle é explorado para evitar que um objetivo seja melhorado em detrimento de outros. O desacoplamento implícito das variáveis de controle é realizado durante os processos de recombinação e mutação. Para extrair a melhor solução compromisso do conjunto Pareto-ótimo utiliza-se um mecanismo de decisão baseado na teoria dos conjuntos fuzzy [11-13]. A eficiência e robustez do AEMO são demonstradas através de resultados obtidos com os sistemas teste IEEE-30 [14], RTS-96 [15] e IEEE-354 [16].

\section{FORMULAÇÃO DO PROBLEMA DE FLUXO DE POTÊNCIA ÓTIMO}

O problema de FPO consiste em determinar um conjunto de variáveis de estado e controle otimizadas da rede elétrica, a partir dos dados de carga e dos parâmetros físicos e topológicos do sistema elétrico de potência. Este problema é caracterizado matematicamente como um problema de otimização não-linear restrito, não-convexo e de grande porte (com milhares de variáveis e restrições), com variáveis contínuas e discretas podendo ser formulado como:

$\operatorname{Min} \sum_{i \in G} C_{2 i}\left(P_{g i}\right)^{2}+C_{1 i}\left(P_{g i}\right)+C_{0 i}$

sujeito a:

$\begin{array}{ll}P_{g i}-P_{L i}-P(\theta, V, t)_{i}=0 & \forall i \in N \\ Q_{g i}-Q_{L i}-Q(\theta, V, t)_{i}+S_{H_{i}}=0 & \forall i \in N \\ P_{g i}^{\min } \leq P_{g i} \leq P_{g i}^{\max } & \forall i \in G \\ Q_{g i}^{\min } \leq Q_{g i} \leq Q_{g i}^{\max } & \forall i \in G \\ V_{i}^{\min } \leq V_{i} \leq V_{i}^{\max } & \forall i \in N \\ \left|S_{i j}\right| \leq S_{i j}^{\max } & \forall i, j \in N \\ t_{i}^{\min } \leq t_{i} \leq t_{i}^{\max } & \forall i \in N \\ S_{H_{i}}^{\min } \leq S_{H_{i}} \leq S_{H_{i}}^{\max } & \forall i \in N\end{array}$

A função objetivo (1) representa o aspecto econômico do sistema elétrico e na prática representa o índice a ser otimizado no despacho econômico, na qual cada unidade geradora é representada por uma curva de custo de geração em função da potência ativa gerada. As restrições de igualdade (2) e (3), também, chamadas de restrições de carga de um problema de $\mathrm{FPO}$, correspondem às equações não-lineares do fluxo de potência clássico e representam a relação entre as variáveis dependentes e as variáveis independentes. As restrições de desigualdade (4)-(9) são incluídas no problema 
para representar os limites físicos dos componentes ou aspectos de segurança relacionados à operação do sistema.

\section{TRATAMENTO DAS RESTRIÇÕES DE DESIGUALDADES E ALGORITMOS EVOLUTIVOS}

A presença de restrições de diversas naturezas introduz uma complexidade adicional para resolver o problema de otimização através dos AEs por dividir o espaço de soluções em duas classes: soluções factíveis (que atendem a todas as restrições simultaneamente) e soluções infactíveis (que violam uma ou mais restrições). Além disso, os conjuntos de soluções factíveis e infactíveis podem ser não-convexos e nãoconectados (ver Fig. 1).

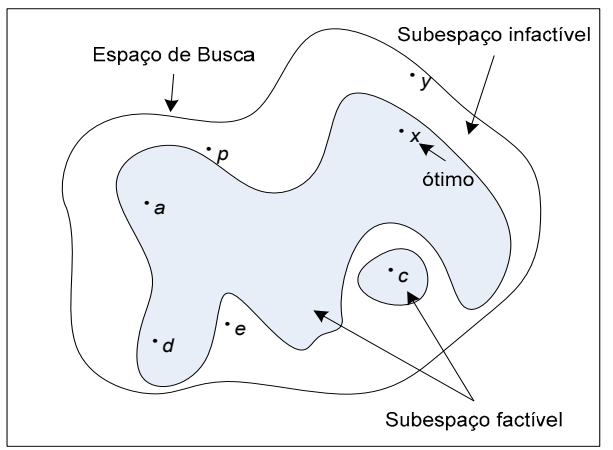

Figura 1: Espaço de busca.

Em sua forma original, todas as restrições pertinentes ao problema de FPO são não-lineares. Entretanto, da mesma forma que na solução do fluxo de potência convencional, a linearização sucessiva das equações envolvidas pode ser aplicada, assim como o desacoplamento físico entre as variáveis referentes à potência ativa - ângulo da tensão e potência reativa - módulo da tensão pode ser vantajosamente explorado. Mas, quanto mais aproximações forem realizadas no modelo matemático menos precisa e longe da realidade será a solução do problema de FPO.

$\mathrm{Na}$ maioria das implementações de AEs para solução do problema de FPO, as restrições violadas são adicionadas à função objetivo do problema através das técnicas de penalidades. Todavia, penalizar as restrições não satisfeitas reduz a aptidão do indivíduo e sua probabilidade de participar do processo de evolução. Por outro lado, é questionável se a penalidade deve impor que todos os indivíduos infactíveis sejam pior que quaisquer outros indivíduos factíveis. Por exemplo, o ponto infactível $y$ na Fig. 1 está muito mais próximo do ponto ótimo $x$ que os pontos factíveis $a, d$ e $c$, conseqüentemente, o ponto $y$ (mesmo sendo infactível) pode fornecer informações mais valiosas se selecionado, mas devido a uma política de penalizar em demasia os pontos infactíveis a probabilidade deste ponto ser selecionado para a recombinação e mutação é muito baixa.

Neste trabalho, para atender as restrições físicas e operacionais, sem comprometer a qualidade das soluções encontradas propõem-se tratar as infactibilidades ocorridas no conjunto de restrições de desigualdade como funções objetivo do problema. Desta forma, o problema (1)-(9) é transformado em um problema e de otimização multiobjetivo e reescrito como segue:

\section{A. Minimização dos custos da geração de potência ativa}

$f_{1}=\operatorname{Min} \sum_{i \in G} C_{2 i}\left(P_{g i}\right)^{2}+C_{1 i}\left(P_{g i}\right)+C_{0 i}$

B. Minimização das violações da capacidade da geração de potência ativa

$h_{1}=\operatorname{Min}\left\{0, P_{g_{\text {ref }}}-P_{g_{\text {ref }}}^{\max }\right\}$

C. Minimização das violações da capacidade da geração de potência reativa

$h_{2}=\operatorname{Min} \sum_{i \in G} \Delta Q_{g i} \quad \forall i \in G$

Em que:

$\Delta Q_{g i}=\left\{\begin{array}{lc}Q_{g i}^{\min }-Q_{g i}, & Q_{g i}<Q_{g i}^{\min } \\ Q_{g i}-Q_{g i}^{\max }, & Q_{g i}>Q_{g i}^{\max } \\ 0, & \text { caso contrário }\end{array}\right.$

D. Minimização das violações nas magnitudes de tensão

$h_{3}=\operatorname{Min} \sum_{i \in N} \Delta V_{i} \quad \forall i \in N$

Em que:

$\Delta V_{i}=\left\{\begin{array}{lr}V_{i}^{\min }-V_{i}, & V_{i}<V_{i}^{\min } \\ V_{i}-V_{i}^{\max }, & V_{i}>V_{i}^{\max } \\ 0, & \text { caso contrário }\end{array}\right.$

E. Minimização das violações nos fluxos de potência nas linhas de transmissão

$h_{4}=\operatorname{Min} \sum_{i, j \in N} \Delta S_{i j} \quad \forall i, j \in N$

Em que:

$$
\Delta S_{i j}=\left\{\begin{array}{lr}
S_{i j}-S_{i j}^{\max }, & S_{i j}>S_{i j}^{\max } \\
0, & \text { caso contrário }
\end{array}\right.
$$

Neste modelo de FPO modificado as restrições são as equações de fluxo de carga (2) e (3)

\section{OTIMIZAÇÃO MULTIOBJETIVO}

Um problema de otimização multiobjetivo é caracterizado pela otimização simultânea de várias funções objetivo com diferentes soluções ótimas [8]. Geralmente, as funções objetivo de um problema de otimização multiobjetivo são nãocomensuráveis e conflitantes entre si e por esta razão não existe uma única solução que seja ótima simultaneamente para todos os objetivos e sim um conjunto de soluções compromisso denominado conjunto eficiente ou Pareto-ótimo. 
Para um problema de otimização multiobjetivo com $N_{o b j}$ funções objetivo $\left(f_{k}\right)$, para serem minimizadas simultaneamente, a solução $x_{1}$ domina a solução $x_{2}$ se as condições abaixo são satisfeitas:

1. $\forall k \in\left\{1,2, \ldots, N_{o b j}\right\}: f_{k}\left(x_{1}\right)<f_{k}\left(x_{2}\right)$

2. $\exists l \in\left\{1,2, \ldots, N_{o b j}\right\}: \quad f_{l}\left(x_{1}\right) \leq f_{l}\left(x_{2}\right)$

Se qualquer uma das condições acima é violada, a solução $x_{1}$ não domina a solução $x_{2}$. Se solução $x_{1}$ domina solução $x_{2}$, então $x_{1}$ é designada solução não-dominada. As soluções que são não-dominadas sobre todo o espaço de busca são chamadas soluções Pareto-ótimas e constituem o conjunto Pareto-ótimo ou a fronteira de Pareto-ótima.

\section{TÉCNICA DE SOLUÇÃO PROPOSTA}

Para solução do FPO tratado como um problema de otimização multiobjetivo propõe-se um AEMO baseado em um procedimento de ordenação dos pontos candidatos a serem pontos eficientes da população [17]. A diferença desta implementação em relação a um algoritmo genético simples está no modo com que o operador de seleção é realizado. Tanto o operador de recombinação quanto o operador de mutação são os usuais dos algoritmos genéticos. Antes do procedimento de seleção ser aplicado, a população é ordenada com base no nível de não-dominância dos indivíduos, isto é, todas as soluções não-dominadas da população corrente recebem valores altos de aptidão. Esta aptidão é a mesma para todos os indivíduos não-dominados, garantindo assim que todos possuam um mesmo potencial reprodutivo.

Para manter a diversidade da população as soluções nãodominadas compartilham os seus valores de aptidão segundo suas distâncias Euclidianas [18]. Finalmente, divide-se o valor da aptidão de cada indivíduo pelo contador de nichos que é proporcional ao número de vizinhos ao seu redor. Este procedimento proporciona a coexistência de pontos ótimos múltiplos na população. O pior valor de aptidão compartilhada na solução da primeira fronteira não-dominada é então armazenado para uso posterior. Depois que o compartilhamento é executado e que as aptidões são modificadas os indivíduos não-dominados são ignorados temporariamente para processar os demais membros da população. O procedimento para determinar novas soluções não-dominadas (segundo nível) é novamente executado, sendo que agora eles recebem um valor de aptidão um pouco menor que o pior valor de aptidão compartilhada no nível anterior. Uma vez mais o procedimento de compartilhamento é executado entre as soluções não-dominadas do segundo nível e as novas aptidões são calculadas como antes. Este processo é continuado até que todos os membros da população tenham um valor de aptidão compartilhado.

Para gerar um conjunto suficientemente diversificado de soluções não-dominadas e obter um bom desempenho, na solução do FPO, o algoritmo proposto combina algumas estratégias, tais como:

- Codificação das variáveis de controle em base real;

- Elitismo;

- Desacoplamento implícito das variáveis do problema;

- Seleção e recombinação simultaneamente;

- Redução do conjunto de Pareto através de clustering;

- Preservação da diversidade.

Adicionalmente, um mecanismo de decisão, baseado na teoria dos conjuntos fuzzy é explorado para extrair a melhor solução compromisso.

\section{A. Codificação e população inicial}

Os indivíduos (cromossomos) que compõem a população são formados por 04 subconjuntos de variáveis, representadas pelas variáveis de controle do problema em questão (ver Fig. 2).

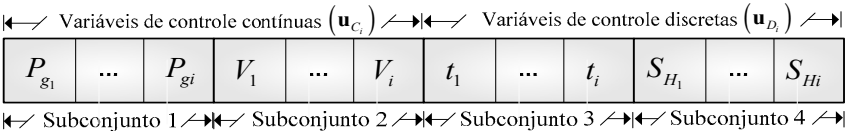
Figura 2: Estrutura do cromossomo da população.

A potência ativa gerada $\left(P_{g i}\right)$ e as magnitudes de tensões $\left(V_{i}\right)$ são tratadas como variáveis de controle contínuas $\left(\mathbf{u}_{C_{i}}\right)$ e geradas de forma pseudoaleatória para atender a região de factibilidade destas variáveis. Os transformadores com controle automático de taps $\left(t_{i}\right)$ e os bancos de capacitores e reatores shunts $\left(S_{H i}\right)$ são representados por valores discretos $\left(\mathbf{u}_{D_{i}}\right)$ como segue:

Seja $n_{i}$ número aleatório inteiro no intervalo $\left[0, \ldots, K_{i}\right]$ e $K_{i}=\operatorname{int}\left(u_{D_{i}}^{\max }-u_{D_{i}}^{\min } / \Delta \mathbf{u}_{D}\right)$, então os valores discretos são dados por:

$u_{D_{i}}=u_{D_{i}}^{\min }+n_{i} \cdot\left(\Delta \mathbf{u}_{D}\right) \quad \forall i \in N$

Tanto as variáveis contínuas, quanto as variáveis discretas são codificadas em base real satisfazendo suas respectivas regiões de factibilidade. As vantagens deste sistema de codificação são armazenar uma maior quantidade de informações que a codificação binária para um cromossomo com a mesma dimensão e trabalhar com a representação real das variáveis do problema.

A partir das variáveis de controle contínuas e discretas estabelecidas para cada indivíduo calculam-se as restrições de fluxo de carga (2) e (3). Estas restrições são resolvidas através do Método de Newton [19] e posteriormente calculam-se as funções objetivo (10)-(14).

\section{B. Estratégia elitista}

O elistimo foi implementado para aumentar desempenho do algoritmo e prevenir a perda das soluções de boa qualidade 
encontradas ao longo da busca. A técnica elitista desenvolvida é baseada em uma população secundária que contém todas as soluções não-dominadas durante as gerações. $\mathrm{Na}$ sua forma mais simples, em cada geração, armazenam-se as soluções não dominadas em um subconjunto elitista $(E)$, para que sejam utilizadas no processo de recombinação com o objetivo de aumentar a pressão de seleção e ao mesmo tempo acelerar a convergência do algoritmo. À medida que o tamanho do subconjunto $E$ aumenta o tempo computacional necessário para esta operação pode ser significativo. Desta forma, em todas as gerações, uma solução não-dominada encontrada na geração corrente só é armazenada se ela for diferente das soluções contidas no subconjunto $E$. Após a inserção de uma solução no subconjunto $E$ realiza-se uma avaliação dos indivíduos através do teste de dominância (eq. 15 e 16) e as soluções que se tornarem dominadas são eliminadas.

\section{Desacoplamento implícito das variáveis de controle}

As violações das restrições de desigualdades são tratadas como funções objetivo do problema e, ao atualizar um determinado conjunto de variáveis de controle através de recombinação ou mutação, um objetivo é melhorado em detrimento de outros. Desta forma, após sortear os indivíduos para executar a recombinação ou mutação, deve-se verificar qual dos objetivos está sendo contemplado e efetuar a recombinação ou mutação considerando apenas $\mathrm{o}(\mathrm{s})$ subconjunto(s) de variáveis de controle que interferem diretamente neste objetivo.

\section{Operadores genéticos}

\section{1) Seleção e recombinação}

O procedimento de seleção adotado é o de torneio, na qual algumas soluções são aleatoriamente escolhidas da população e, com base em algum critério, a solução vencedora é então selecionada. O procedimento de torneio é efetuado diretamente sobre as ordens (fronteiras) recebidas pelos indivíduos, deste modo, os indivíduos são selecionados não só pelas suas aptidões, mas sim pelas suas aptidões dentro do contexto multiobjetivo de dominância. Além disso, este procedimento é realizado em conjunto com o operador de recombinação de um único ponto.

Seja $\left\{N_{p o p}\right\}$ o número máximo de indivíduos da população e $\{M\}$ um conjunto que contém as soluções $\left\{M / M \in N_{p o p}\right.$ e $\left.M \notin E\right\}$ a serem utilizadas nos processos de seleção e recombinação, como segue:

i. Selecionar por torneio um indivíduo pai, $\mathrm{P} 1$, do subconjunto $M$;

ii. Selecionar aleatoriamente o segundo pai, P2, do subconjunto elitista $E$;

iii. Selecionar aleatoriamente um dos objetivos do problema e iniciar o processo de recombinação;

$i v$. Gerar um número aleatório $r \in[0,1]$. Se $r<\operatorname{Pr}(\operatorname{Pr}$ é a probabilidade de recombinação), então obter aleatoriamente $\mathrm{o}$ ponto de recombinação. Caso contrário, voltar ao passo $i$;

v. Se o objetivo escolhido no passo iii se referir ao custo da geração, então a recombinação de um único ponto será realizada considerando todos os subconjuntos de variáveis de controle (Fig. 2). Caso contrário, o desacoplamento implícito das variáveis do problema será realizado;

$v i$. Repetir os passos de $i$ à $v$ até que a nova população possua o número de indivíduos predefinido.

\section{2) Mutação}

A mutação é um operador de grande importância para a solução de problema do mundo real, pois introduz, aleatoriamente, novas informações na população, prevenindo a convergência prematura do algoritmo. A seqüência deste procedimento é descrita abaixo:

i. Gerar um número aleatório $m \in[0,1]$;

ii. Se $m<\operatorname{Pm}$ (Pm é a probabilidade de mutação), então selecionar aleatoriamente um dos objetivos do problema, para realizar a mutação. Caso contrário, voltar ao passo $i$

iii. Se o objetivo escolhido no passo $i i$ for diferente do custo da geração, então deve-se realizar o desacoplamento implícito das variáveis do problema. Caso contrário, a mutação será efetuada em todos os subconjuntos das variáveis de controle (Fig. 2);

iv. Selecionar o ponto de mutação para a variável que sofrerá mutação;

v. Trocar o valor atual da variável selecionada por um valor gerado, aleatoriamente, no domínio desta variável;

$v i$. Repetir os passos $i$ à $v$ até que a nova população tenha o número de indivíduos predefinido.

\section{E. Redução do Conjunto de Pareto}

Em alguns problemas o conjunto Pareto-ótimo pode ser extremamente grande. Nestes casos, a redução do conjunto de soluções não-dominadas sem a degradação das características da fronteira Pareto-ótimo é indispensável. Para a redução do número de soluções contidas no conjunto Pareto-ótimo utilizou-se a técnica denominada clustering [20]. Esta técnica particiona o conjunto de soluções dominantes em $n$ grupos (clusters) de acordo com a proximidade das soluções. Para cada cluster, seleciona-se uma solução representativa (centróide) e as soluções restantes são descartadas. A distância entre quaisquer dois elementos do conjunto de soluções nãodominadas é encontrada através da distância euclidiana [18].

\section{F. Preservação da Diversidade}

Geralmente para preservar a diversidade da população em algoritmos evolutivos multiobjetivos utiliza-se a técnica de niching [9]. A técnica de niching consiste na divisão da população em espécies para reduzir a competição por recursos 
e criar subpopulações estáveis, cada uma delas concentrada em um nicho do espaço de busca. O esquema de niching utiliza um mecanismo de sharing que trabalha alterando a função de avaliação de cada elemento da população de acordo com o número de indivíduos semelhantes a ele na população [21]. Porém, ao contrário do esperado, seus resultados não melhoraram significativamente a diversidade das soluções não-dominadas encontradas. Dessa forma, para preservar a diversidade na população, além do mecanismo de sharing utilizaram-se as taxas de recombinação $(P r)$ e mutação $(P m)$ atualizadas de forma adaptativa, como segue:

$$
\begin{aligned}
& P r=\left[P r^{\max }-i g \cdot\left(P r^{\max }-P r^{\min }\right)\right] / \text { nmax } \\
& P m=\left[P m^{\text {mim }}+i g \cdot\left(P m^{\max }-P m^{\min }\right)\right] / \text { nmax }
\end{aligned}
$$

\section{G. Melhor solução compromisso}

Para determinar uma solução particular, após a convergência do algoritmo aplica-se um mecanismo de decisão, baseado na teoria dos conjuntos fuzzy. Os conjuntos fuzzy são definidos através de equações denominadas funções de pertinência $\left(\mu_{i}\right)$. Estas funções representam o grau de pertinência no conjunto fuzzy usando valores 0 e 1 . Os valores das funções de pertinência indicam o grau de satisfação das funções objetivos do problema. A equação abaixo expressa a função de pertinência que é definida como em [12].

$$
\mu_{i}= \begin{cases}1 & f_{i}(x) \leq f_{i}^{\min }(x) \\ 0 & f_{i}(x) \geq f_{i}^{\max }(x) \\ \frac{f_{i}^{\max }(x)-f_{i}(x)}{f_{i}^{\max }(x)-f_{i}^{\min }(x)} & f_{i}^{\min }(x) \leq f_{i}(x) \leq f_{i}^{\max }(x) \\ & i=1, \ldots N_{o b j}\end{cases}
$$

Seja $N_{d o m}$ o número de soluções não-dominadas da primeira fronteira de Pareto, então, para cada solução nãodominada $k\left(k \in N_{d o m}\right)$ a função de pertinência é normalizada como:

$$
\mu^{k}=\frac{\sum_{i=1}^{N o b j} \mu_{i}^{k}}{\sum_{k=1}^{N_{d o m}} \sum_{i=1}^{N o b j} \mu_{i}^{k}}
$$

A melhor solução compromisso é dada por $\gamma=\max \left\{\mu^{k}\right\}, k=1,2, \ldots, N_{d o m}$.

\section{RESUlTADOS E DISCUSSÕES}

Para investigar a eficiência do AEMO na solução do problema de FPO apresentam-se os resultados obtidos com os sistemas teste IEEE-30, RTS-96 e IEEE-354. A eficiência e robustez do AEMO são avaliadas utilizando-se três diferentes testes:

Teste 1) Despacho econômico;
Teste 2) Despacho econômico e minimização das perdas nas linhas de transmissão;

Teste 3) Minimização do custo de operação, das perdas nas linhas de transmissão e emissão de gases poluentes.

As simulações realizadas têm como objetivo testar o desempenho e a robustez da metodologia em relação aos seguintes aspectos:

- Minimizar o custo de geração;

- Atender os limites físicos dos componentes e os aspectos de segurança relacionados à operação do sistema;

- Eficiência na solução de problemas multiobjetivos;

- Estabelecer uma análise comparativa entre o AEMO proposto e uma técnica de otimização clássica.

Nas simulações com os três sistemas teste adotaram-se os seguintes limites e parâmetros:

- Limites mínimos e máximos das taxas recombinação e mutação são $P r^{\min }=0,001, P r^{\max }=0,9, P m^{\min }=0,01 \mathrm{e}$ $P m^{\text {max }}=0,5$, respectivamente;

- Limites mínimo e máximo para os transformadores com controle automático de taps são $0,9 \quad p u$ e $1,1 \quad p u$, respectivamente,

- Limites mínimo e máximo para os bancos de capacitores/reatores shunt são 0 e valor encontrado no banco de dados (caso base), respectivamente;

- Tamanho do passo para a discretização dos transformadores taps é $\Delta \mathbf{u}_{D}=0,01 p u$;

- Tamanho do passo para a discretização dos bancos de capacitores/reatores shunt são $\Delta \mathbf{u}_{D}=0,02 p u$ (sistema IEEE-30) e $\Delta \mathbf{u}_{D}=0,125 p u$ (sistemas RTS-96 e IEEE354).

Inicialmente, as funções objetivo consideradas são otimizadas individualmente para obter os pontos extremos da curva de Pareto. Para as funções objetivo referentes às restrições de desigualdade os valores mínimos são considerados iguais a zero e os valores máximos são iguais a soma das infactibilidades obtidas nas simulações do fluxo de carga convencional (método de Newton).

A análise comparativa é realizada considerando-se o sistema teste IEEE-30 e utilizando-se o software MATPOWER [22]. Este software resolve o problema de despacho econômico através de técnica de programação linear sucessiva.

\section{A. Sistema IEEE-30 barras}

Este sistema possui 06 geradores, 04 transformadores e 02 capacitores shunts. O limite mínimo da magnitude tensão é $0,95 p u$ e os limites máximos são $1,10 p u$ para as barras de geração $(2,5,8,11$ e 13) e 1,05 pu para as demais barras. Na Tabela I apresentam-se os dados necessários para a análise do despacho econômico. 
TABELA I

DADOS DOS GERADORES.

\begin{tabular}{|c|c|c|c|c|c|c|}
\hline \multicolumn{2}{|c|}{ Geradores } & \multicolumn{2}{|c|}{$C_{2 i}\left(P_{g i}\right)^{2}+C_{1 i}\left(P_{g i}\right)+C_{0 i}$} & \multirow{2}{*}{$\frac{(\$ / \mathrm{h})}{C_{0 i}}$} & \multirow{2}{*}{$\begin{array}{c}P_{g i}^{\max } \\
(M W)\end{array}$} & \multirow{2}{*}{$\begin{array}{c}P_{g i}^{\min } \\
(M W)\end{array}$} \\
\hline $\mathrm{N}^{0}$ & Barra & $C_{2 i}$ & $C_{1 i}$ & & & \\
\hline 1 & 1 & 10 & 200 & 100 & 150 & 5 \\
\hline 2 & 2 & 10 & 150 & 120 & 150 & 5 \\
\hline 3 & 5 & 20 & 180 & 40 & 150 & 5 \\
\hline 4 & 8 & 10 & 100 & 60 & 150 & 5 \\
\hline 5 & 11 & 20 & 180 & 40 & 150 & 5 \\
\hline 6 & 13 & 10 & 150 & 100 & 150 & 5 \\
\hline
\end{tabular}

Os resultados referentes à melhor solução compromisso obtidos com a aplicação do AEMO são apresentados nas Tabelas II, III e IV. Nestas tabelas exibem-se os resultados numéricos das simulações para os três testes em análise. Nas Figuras 3 e 4 apresentam-se a fronteira de Pareto-ótima e a melhor solução compromisso obtidas nas simulações com os Testes 2 e 3, respectivamente. Na Tabela V apresentam-se os resultados de simulação obtidos através do software MATPOWER

TABELA II

MELHOR SOLUÇÃO COMPROMISSO - IEEE-30.

\begin{tabular}{|c|c|c|c|}
\hline \multirow{2}{*}{ Geradores } & \multicolumn{3}{|c|}{ Potência ativa gerada $(\boldsymbol{M W})$} \\
\cline { 2 - 4 } & Teste 1 & Teste 2 & Teste 3 \\
\hline$P_{g 1}$ & 29,11 & 27,92 & 31,34 \\
$P_{g 2}$ & 24,84 & 26,36 & 26,28 \\
$P_{g 5}$ & 75,32 & 76,50 & 75,86 \\
$P_{g 8}$ & 50,12 & 51,51 & 50,57 \\
$P_{g 11}$ & 74,04 & 71,66 & 72,15 \\
$P_{g 13}$ & 31,75 & 31,12 & 28,87 \\
\hline Custo (\$/h) & $\mathbf{9 0 2 8 3 5 , 2 3}$ & $\mathbf{9 0 2 9 2 8 , 7 5}$ & $\mathbf{9 0 2 5 8 0 , 4 3}$ \\
Perdas (MW) & $\mathbf{1 , 6 9}$ & $\mathbf{1 , 6 7}$ & $\mathbf{1 , 6 8}$ \\
Emissão (ton/h) & $\mathbf{0 , 2 0 5}$ & $\mathbf{0 , 2 0 5}$ & $\mathbf{0 , 2 0 4}$ \\
Tempo Comp. (s) & $\mathbf{3 , 2 7}$ & $\mathbf{3 , 6 3}$ & $\mathbf{3 , 4 2}$ \\
\hline
\end{tabular}

TABELA III

AJUSTES DE TAPS DOS TRANSFORMADORES ( $p u$ ) - IEEE-30

\begin{tabular}{|c|c|c|c|c|}
\hline Taps & Linha & Teste 1 & Teste 2 & Teste 3 \\
\hline$t_{1}$ & $6-9$ & 1,04 & 1,04 & 0,99 \\
$t_{2}$ & $6-10$ & 0,99 & 1,09 & 1,02 \\
$t_{3}$ & $4-12$ & 1,01 & 1,07 & 1,00 \\
$t_{4}$ & $28-27$ & 0,98 & 0,93 & 0,98 \\
\hline
\end{tabular}

TABELA IV

AJUSTE DOS BANCOS DE CAPACITORES/REATORES SHUNTS $(p u)$ - IEEE-30

\begin{tabular}{|c|c|c|c|}
\hline Barra & Teste 1 & Teste 2 & Teste 3 \\
\hline 10 & 0,140 & 0,190 & 0,190 \\
24 & 0,043 & 0,043 & 0,043 \\
\hline
\end{tabular}

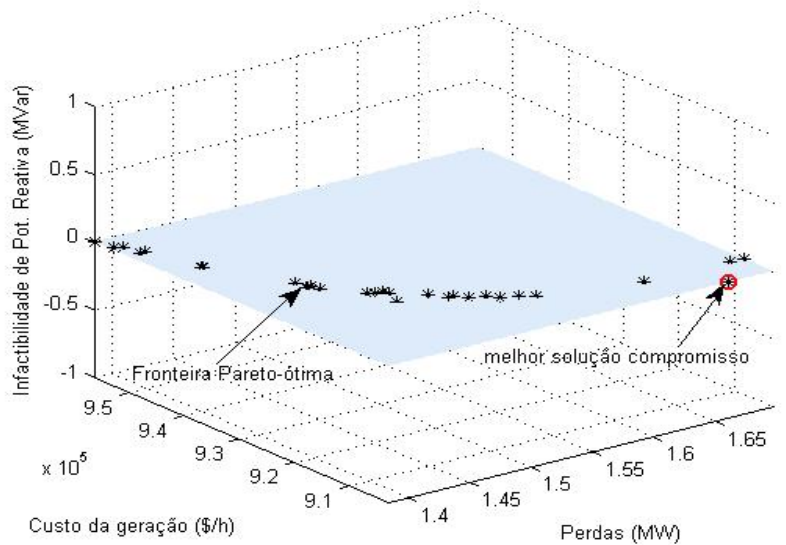

Figura 3: Fronteira de Pareto-ótima e melhor solução compromisso - Teste 2.

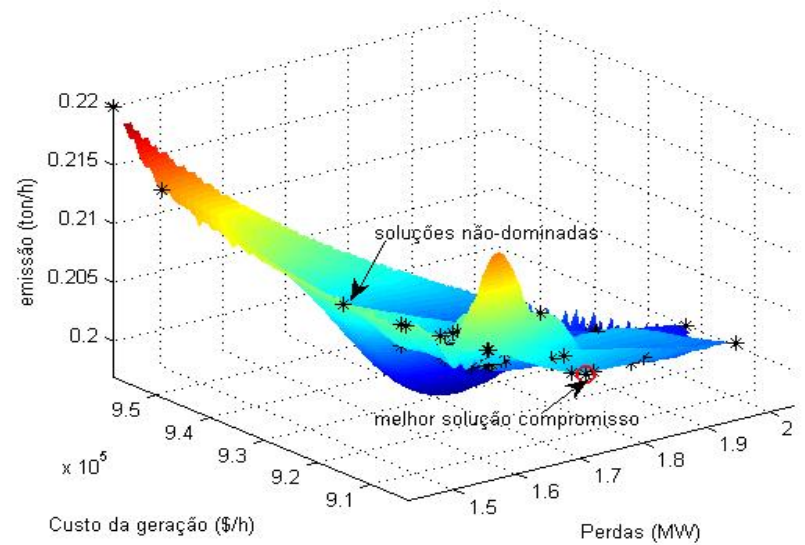

Figura 4: Fronteira de Pareto-ótima e melhor solução compromisso - Teste 3.

TABELA V

RESULTADOS OBTIDOS COM O MATPOWER - IEEE-30

\begin{tabular}{|c|c|c|}
\hline \multicolumn{3}{|c|}{ Potência ativa gerada $(M W)$} \\
\hline$P_{g 1}: 30,87$ & $P_{g 5}: 79,19$ & $P_{g 11}: 64,87$ \\
\hline$P_{g 2}: 26,12$ & $P_{g 8}: 52,70$ & $P_{g 13}: 31,36$ \\
\hline \multicolumn{3}{|c|}{ Custo: $\quad 907351,56(\$ / h)$} \\
\hline \multicolumn{3}{|c|}{ Perdas: $1,70 \mathrm{MW}$} \\
\hline \multicolumn{3}{|c|}{ Tempo Computacional: $\quad 2,66 \mathrm{~s}$} \\
\hline
\end{tabular}

\section{B. Sistema RTS-96}

O sistema RTS-96 consiste de 03 áreas interconectadas através de 05 linhas de ligação. Este sistema possui 73 barras, 120 linhas de transmissão, 33 geradores, 15 transformadores com ajuste automático de taps e 03 capacitores/reatores shunts. Os limites mínimo e máximo adotados para as magnitudes de tensão são 0,95 pu e 1,05 pu. Na Tabela VI apresentam-se os resultados numéricos obtidos com os Testes 1 e 2. A fronteira de Pareto-ótima e a melhor solução compromisso obtida com o Teste 2 é apresentada na Fig. 5. 
TABELA VI

MELHOR SOLUÇÃO COMPROMISSO.

\begin{tabular}{|c|c|c|}
\hline \multirow{2}{*}{ Áreas } & \multicolumn{2}{|c|}{ Custo de Geração (\$/h) } \\
\hline & Teste 1 & Teste 2 \\
\hline área 1 & 37520,66 & 38273,92 \\
\hline área 2 & 43318,99 & 44483,47 \\
\hline área 3 & 44077,99 & 43784,47 \\
\hline Custo Total(\$/h) & 124916,65 & 126541,86 \\
\hline Perdas (MW) & 138,35 & 110,03 \\
\hline Tempo Computacional & $2 \min \mathrm{e} 48 \mathrm{~s}$ & $2 \min$ e $57 \mathrm{~s}$ \\
\hline
\end{tabular}

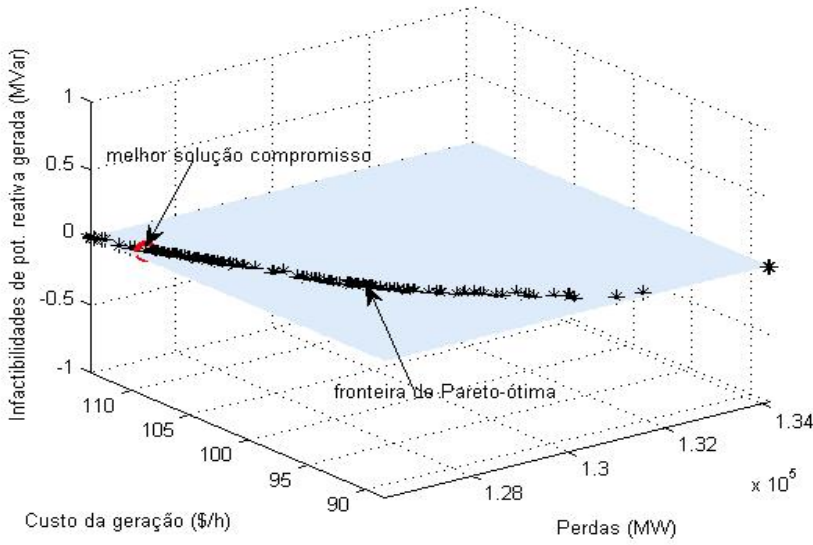

Figura 5: Fronteira de Pareto-ótima e melhor solução compromisso - Teste 2.

\section{Sistema IEEE-354}

O sistema IEEE-354 é baseado no sistema teste IEEE-118 [23] e consiste de 03 áreas interconectadas por 06 linhas de ligação. Este sistema possui 354 barras, 164 geradores, 558 linhas de transmissão, 42 capacitores/reatores shunts e 27 transformadores com controle automático de taps.

Os limites mínimos e máximos adotados para as magnitudes de tensão são $0,96 p u$ e 1,04 pu, respectivamente.

Na Tabela VII são mostrados os resultados obtidos para os Testes 1 e 2. Na Fig. 6 pode-se observar a fronteira de Paretoótima, considerando se a redução nos custos das perdas nas linhas de transmissão, o custo total da geração de potência ativa e a minimização das infactibilidades ocorridas na geração de potência reativa.

TABELA VII

MELHOR SOLUC̃̃̃ COMPROMISSO - IEEE-354.

\begin{tabular}{|l|c|c|}
\hline \multicolumn{3}{|c|}{ Custo da geração (\$/h) } \\
\hline Áreas & Teste 1 & Teste 2 \\
\hline Área 1 & 133443,14 & 134377,78 \\
Área 2 & 128448,28 & 132486,57 \\
Área 3 & 129811,37 & 136281,53 \\
\hline Custo Total & $\mathbf{3 9 1 7 0 2 , 7 9}$ & $\mathbf{4 0 3 1 4 5 , 5 3}$ \\
Perdas (MW) & $\mathbf{2 7 0 , 3 3}$ & $\mathbf{1 5 9 , 3 8}$ \\
Tempo Comp. & $\mathbf{3 h}$ e 29 min & $\mathbf{3 h}$ e 37 min \\
\hline
\end{tabular}

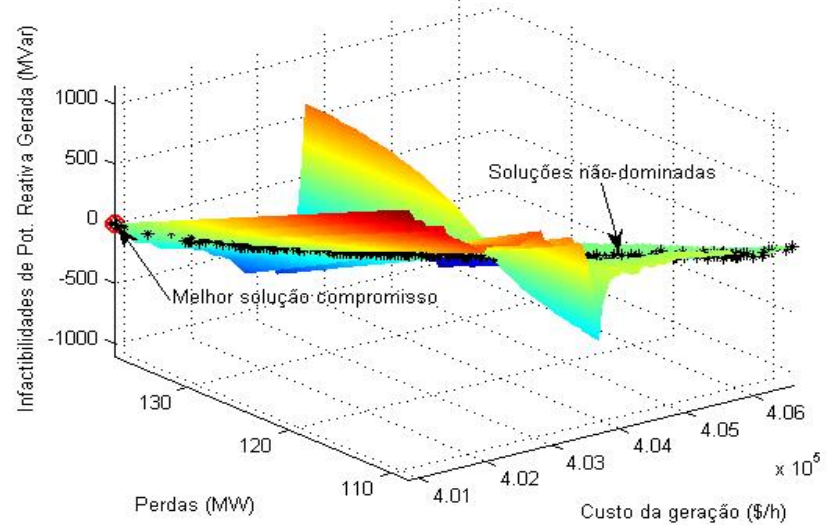

Figura 6: Fronteira de Pareto-ótima e melhor solução compromisso - Teste 2.

\section{Análise dos resultados}

O AEMO implementado convergiu para soluções de boa qualidade, ou seja, alteraram-se os controles de potência reativa disponíveis, melhorando o perfil de tensão. Ressalta-se que as magnitudes de tensões permaneceram dentro dos limites estabelecidos em todos os casos analisado. As restrições referentes aos fluxos de potência nas linhas de transmissão e à capacidade de geração de potência ativa foram devidamente atendidas em todas as simulações realizadas. A restrição de desigualdade referente à geração de potência reativa foi devidamente atendida. Este fato pode ser verificado observando-se as figuras 3, 4,5 e 6 .

Na Tabela VIII apresentam-se os resultados obtidos através das simulações com o fluxo de carga convencional (FCC) para os sistemas teste RTS-96 e IEEE-118. Observando-se as infactibilidades na geração de potência reativa conclui-se que o sistema teste IEEE-354 tem excesso de reativo. Conseqüentemente, devido a esta característica, nas simulações com este sistema teste, para atender a restrição referente à geração de potência reativa foram necessários 1600 gerações e 900 indivíduos, acarretando em um elevado tempo computacional.

Salienta-se que não foi realizado o Teste 3 para os sistemas teste RTS-96 e IEEE-354 devido à falta de dados referentes aos coeficientes de emissão de gases dos geradores.

Comparando-se os resultados apresentados nas Tabelas II e $\mathrm{V}$ observa-se que os resultados obtidos com o AEMO são melhores e mais realistas. No entanto, a limitação do AEMO é o tempo esforço computacional exigido por estes algoritmos, o que ainda dificulta a aplicação dos mesmos para o planejamento de curto prazo e controle em tempo real.

TABELA VIII

Resultados do fluxo de carga convencional.

\begin{tabular}{|l|r|r|}
\hline & RTS-96 & \multicolumn{1}{|c|}{ IEEE-354 } \\
\hline Custo Total (\$/h) & 132171,40 & 394019,10 \\
Perdas (MW) & 117,45 & 409,76 \\
Infac. na geração de & 116,79 & 1109,80 \\
potência reativa (MVar) & & \\
\hline
\end{tabular}




\section{CONCLUSÕES}

Neste trabalho o problema de despacho econômico foi abordado como um problema de otimização multiobjetivo e resolvido através de um algoritmo evolutivo baseado na teoria de Pareto. Os resultados demonstram que a metodologia proposta é eficiente para resolver problemas de otimização multiobjetivos e mono-objetivo que envolve um grande número de variáveis discretas e várias restrições. As soluções não-dominadas na fronteira de Pareto são bem distribuídas e apresentam diversidade satisfatória. Além disso, a metodologia não impõe nenhuma limitação quanto ao número de funções objetivo, sendo assim, o aumento do número de funções objetivo, tais como, emissão de gases poluentes e segurança podem ser consideradas de forma simples e eficiente na metodologia proposta.

\section{REFERÊNCIAS}

[1] H. W Dommel e W. F. Tinney, “Optimal power flow solutions;” IEEE Trans. on Power Apparatus and Systems, no. 87, pp. 1866-1876, Oct. 1968.

[2] J. B. Stott e E. Hobson, "Power system security control calculation using linear programming;" IEEE Trans. Power Aparrat. Syst., pt. I and II, vol. PAS-97, pp.1713-1731, Sept/Oct. 1978.

[3] S. Granville, "Optimal reactive dispatch through interior point methods," IEEE Trans. Power Syst., vol. 9, no. 1, pp. 136-146, Feb. 1994.

[4] A. Momoh; M. E. El-Hawary e R. Adapa, "A review of selected optimal power flow literature to 1993;" IEEE Trans. Power Syst., pt. I and II, vol. 14, pp. 96-111, Feb. 1999.

[5] D. Goldberg e J. Richardson, "Genetic algorithms with sharing for multimodal function optimization;" Genetic algorithms and their applications: Proceedings of the Second International Conference on Genetic Algorithms, 1989.

[6] D. Lenive, "Genetic algorithms: a practitioner's view;" Journal on Computing, vol. 9, no. 3, pp. 256-259, 1997.

[7] C. R. Reeves, "Genetic algorithms for the operations researcher;" Journal on Computing, vol. 9, no. 3, pp. 231-250, 1997.

[8] K. Deb; A. Pratap; S. Agarwal e T.Meyarivan, "A fast and elitist non dominated sorting genetic algorithm for multiobjective optimization: NSGA-II;" Kan Gal report, 2000.

[9] E. A. Amorim; F. G. M. Lima; R. Romero; J. R. S. Mantovani, "Multiarea optimal power flow using multiobjective evolutionary Algorithm;" In: 2009 IEEE PES - General Meeting, 2009, Calgary. Anais do. USA : IEEE - Power \& Energy Society, p. 01-08, 2009.

[10] E. A. Amorim; F. G. M. Lima; S. H. M. Hashimoto; J. R. S. Mantovani, "Fluxo de Potência Ótimo Descentralizado Utilizando Algoritmos Evolutivos Multiobjetivo;" SBA. Sociedade Brasileira de Automática, v. 20, p. 217-132, 2009.

[11] M. A.Abido, "Multiobjective evolutionary algorithms for electric power dispatch problem;" IEEE Trans. On Evolutionary Computations, vol. 10, no. 3, pp. 315-329, June 2006.

[12] J. S. Dhillon; S. C. Parti e D. P. Kothari, "Fuzzy decision making in stochastic multiobjective short-term hydrothermal scheduling," IEE Proc.-C, 149, vol.2, pp. 191-200, Mar. 2002.

[13] J. S. Dhillon; S. C. Parti e D. P. Kothari, "Stochastic economic emission load dispatch," Electric Power Syst. Res., vol. 26, pp. 186$197,1993$.

[14] O. Alsac e B. Stott, "Optimal load flow with steady state security;" IEEE Trans. Power Syst., vol. PAS-93, pp. 745-751, May. 1974.

[15] C. Grigg; P. Wong; P. Albrecht; R. Allan; M. Bhavaraju; R. Billinton; Q. Chen; C. Fong; S. Haddad; S. Kuruganty; W. Li ; R. Mukerji; D.Patton; N. Rau, D. Reppen, A. Schneider, M. Shahidehpour, C., Singh, "The reliability test system-1996;" IEEE Trans. Power Syst, Vol. 14, pp.1010-1020, Aug. 1999.

[16] J. A. Aguado; V. H. Quintana e A. J. Conejo, "Optimal power flows of interconnected power systems;" in IEEE Power Eng. Soc. Summer Meeting, vol. 2, pp. 814-819, 1999.
[17] N. Srinivas e K. Deb, "Multi-Objective Function Optimization Using Non-Dominated Sorting Genetic Algorithm;" Evolutionary Computation, 2(3), pp. 221-248, 1995.

[18] E. Zitzler e L. Thiele, "Multiobjective evolutionary algorithm: a comparative case study and the Strength Pareto approach;" IEEE Transaction on Evolutionary Computation, vol. 3, no. 4, pp. 257-271, 1999.

[19] A. Monticelli, "Fluxo de Carga em Redes de Energia Elétrica;" Editora Edgard Blücher Ltda, 1983.

[20] J. N. Morse, "Reducing the size of nondominated set: Pruning by clutering;" Comput. Oper. Res., vol. 7, no. 1-2, pp.55-66, 1980.

[21] S. W. Mathfound, "Niching methods, In: Back, T.; Fogel, D.B.; Michalewicz (Eds.)," Evolutionary Computation 2, pp 87-92, Institute of Physics Publishing, 2000.

[22] R. Zimmerman e D. Gan, MATPOWER, http://www.pserc.cornell.edu/powerweb/, 1997.

[23] W. M. Lebow; R. K. Mehra; R. Nadira; R. Rouhani, e P.B. Usoro, "Optimization of Reactive Volt-Amperes (VAR) Sources in System Planning" EPRI Report El-3729, vol I, Project 2109-1, 1984.

[24] K. W. Rodrigues Coutinho Braga de Oliveira, N. Teixeira do Nascimento, O. R. Saavedra, "An Evolutionary Approach for the Solution of the Economic Dispatch Considering Generation Constraints", IEEE LATIN AMERICA TRANSACTIONS, Vol. 6, No. 1, pp. 42-50, March 2008.

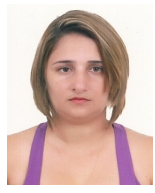

Elizete de Andrade Amorim possui graduação em Matemática pela Universidade Federal de Mato Grosso do Sul - UFMS, campus de Três Lagoas (1997) mestrado e doutorado em Engenharia Elétrica pela Universidade Estadual Paulista Júlio de Mesquita Filho (UNESP) - Campus de Ilha Solteira (2001 e 2006). Atualmente é professora pesquisadora do CNPq na UFMS - Universidade Federal de Mato Grosso do Sul (departamento de engenharia elétrica - Campo Grande/MS). Tem interesse e desenvolve pesquisas nas áreas de Planejamento e Controle de Sistemas de Energia Elétrica, atuando principalmente nos seguintes temas: Desenvolvimento de modelos matemáticos e aplicação de técnicas de otimização clássica e metaheurísticas em problemas de planejamento e operação de sistemas elétricos de potência.

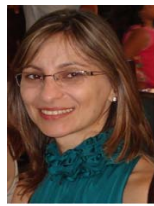

Selma H. M. Hashimoto possui graduação em Matemática pela UFMS, campus de Três Lagoas (1997) mestrado e doutorado em Engenharia Elétrica pela Universidade Estadual Paulista Júlio de Mesquita Filho (UNESP) - Campus de Ilha Solteira (1999 e 2005). Atualmente é professora pesquisadora do CNPq na UFMS - Universidade Federal de Mato Grosso do Sul (departamento de engenharia elétrica Campo Grande/MS).

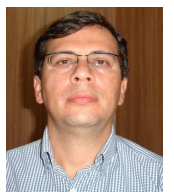

Flávio Guilherme de Melo Lima possui graduação em Engenharia Elétrica pela Universidade Federal do Ceará (1990), mestrado e doutorado em Engenharia Elétrica pela Universidade Estadual de Campinas (1993 e 2000). Atualmente é professor do Departamento de Engenharia Elétrica da Universidade Federal de Mato Grosso do Sul (UFMS). Tem interesse e desenvolve pesquisas nas áreas de planejamento e operação de sistemas elétricos.

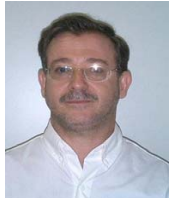

José Roberto Sanches Mantovani possui graduação em Engenharia Elétrica pela Universidade Estadual Paulista Júlio de Mesquita Filho (UNESP) - Campus de Ilha Solteira (1981), mestrado e doutorado em Engenharia Elétrica pela Faculdade de Engenharia Elétrica e de Computação da Universidade Estadual de Campinas (1987 e 1995). Atualmente é professor adjunto do Departamento de Engenharia Elétrica da FEIS-UNESP. Tem interesse e desenvolve pesquisas nas áreas de Planejamento e Controle de Sistemas de Energia Elétrica, atuando principalmente nos seguintes temas: Desenvolvimento de modelos matemáticos e aplicação de técnicas de otimização clássica e metaheurísticas em problemas de planejamento e controle de sistemas de geração, transmissão e distribuição de energia elétrica, confiabilidade e qualidade do fornecimento de energia de sistemas de distribuição de energia elétrica. 